\title{
Wave packet dynamics in semiconductor quantum rings of finite width
}

\author{
Andrey Chaves, ${ }^{1, *}$ G. A. Farias, ${ }^{1, \dagger}$ F. M. Peeters, ${ }^{1,2, \$}$ and B. Szafran ${ }^{3}$ \\ ${ }^{1}$ Departamento de Física, Universidade Federal do Ceará, Caixa Postal 6030, Campus do Pici, 60455-900 Fortaleza, Ceará, Brazil \\ ${ }^{2}$ Department of Physics, University of Antwerp, Groenenborgerlaan 171, B-2020 Antwerp, Belgium \\ ${ }^{3}$ Faculty of Physics and Applied Computer Science, AGH University of Science and Technology, al. Mickiewicza 30, \\ 30-059 Kraków, Poland \\ (Received 27 July 2009; revised manuscript received 20 August 2009; published 25 September 2009)
}

\begin{abstract}
The time evolution of a wave packet injected into a semiconductor quantum ring is investigated in order to obtain the transmission and reflection probabilities. Within the effective-mass approximation, the timedependent Schrödinger equation is solved for a system with nonzero width of the ring and leads and finite potential-barrier heights, where we include smooth lead-ring connections. In the absence of a magnetic field, an analysis of the projection of the wave function over the different subband states shows that when the injected wave packet is within a single subband, the junction can scatter this wave packet into different subbands but remarkably at the second junction the wave packet is scattered back into the subband state of the incoming wave packet. If a magnetic field is applied perpendicularly to the ring plane, transmission and reflection probabilities exhibit Aharonov-Bohm (AB) oscillations and the outgoing electrons may end up in different subband states from those of the incoming electrons. Localized impurities, placed in the ring arms, influence the $\mathrm{AB}$ oscillation period and amplitude. For a single impurity or potential barrier of sufficiently strong strength, the period of the $\mathrm{AB}$ oscillations is halved while for two impurities localized in diametrically opposite points of the ring, the original $\mathrm{AB}$ period is recovered. A theoretical investigation of the confined states and time evolution of wave packets in $\mathrm{T}$ wires is also made, where a comparison between this system and the lead-ring junction is drawn.
\end{abstract}

DOI: 10.1103/PhysRevB.80.125331

PACS number(s): 73.21.Hb, 73.63.Nm, 73.43.Cd

\section{INTRODUCTION}

Aharonov-Bohm (AB) (Ref. 1) effect is a fundamental problem at the heart of quantum mechanics. This effect can be observed in an interference experiment, e.g., as an oscillating current flowing through a loop that is threaded by a magnetic flux. It was first observed in a metal ring ${ }^{2}$ and subsequently in a two-dimensional electron-gas ring ${ }^{3}$ and has been intensively studied over the years both experimentally and theoretically. Due to recent advances in growth of semiconductor structures and nanolithography it has become possible to fabricate well-defined nanoring structures of high quality with controllable dimensions. ${ }^{4}$ Recently, Martins et $a l^{5}$ combined scanning gate microscopy experiments and simulations to demonstrate low-temperature imaging of the electron probability density in InGaAs/InAlAs quantum rings. It was also shown that a tip-induced modulation of the conductance arises from electron wave-function interferences. A theoretical analysis of these AB rings was made later, by Pala et al., ${ }^{6}$ where a generalized Kramers-Kronig relation was demonstrated between the local density of states and the conductance variation due to the scanning tip. Van der Wiel et al. ${ }^{7}$ have studied the AB effect in a mesoscopic semiconductor ring with tunnel barriers defined by metallic gates on each ring arm, which enabled them to apply a welldefined potential difference between the two halves of the ring. By tuning the voltage, they were able to control the amplitude and phase of the $\mathrm{AB}$ oscillations in the conductance. Interference effects of single electrons in a solid-state environment were also observed experimentally by Gustavsson et al., ${ }^{8}$ the time-resolved detection of electrons passing through a double quantum dot embedded in an Aharonov-
Bohm interferometer was demonstrated, where it was observed that, even though the electrons travel one-by-one through the system, a well-pronounced $\mathrm{AB}$ interference is still built up.

In a previous theoretical work by Szafran and Peeters, ${ }^{9}$ it was shown that the Lorentz force leads to an asymmetric injection of the electrons into the ring arms which resulted in a smaller interference effect and, consequently, to a reduction in the amplitude of the AB oscillations. ${ }^{10}$ In Refs. 9 and 10, the leads and the ring were effectively one dimensional, i.e., the magnetic field cannot deflect the electron wave functions in the leads and the ring, and the Lorentz deflection is only active in the junction region. Furthermore, the electron was locked in the lowest subband and higher subbands were not taken into account.

In the present paper, we extend Refs. 9 and 10 as follows: (1) we include the finite width of the leads and ring, (2) we do not limit the number of subbands and, therefore, allow transitions between subbands, and (3) the lead-ring connection is modeled more realistically, i.e., the broadening of the junction is included, which is unavoidable in a real experiment. We found that this adiabatic connection between the leads and the ring has several consequences: (i) it influences strongly the injection efficiency from the lead into the ring, (ii) it leads to additional interferences, (iii) it acts as a scattering center inducing transitions between the different subbands, and (iv) it leads to localized and resonant states in the junction. The finite width of the ring and the leads results in subband selection effects which are absent in the pure onedimensional models. Although most of the experiments up to now consider rings with typical radii and widths of order 100 $\mathrm{nm}$, the present work is focused on narrower systems, where the separation between subbands is larger, in order to get a 
(a)

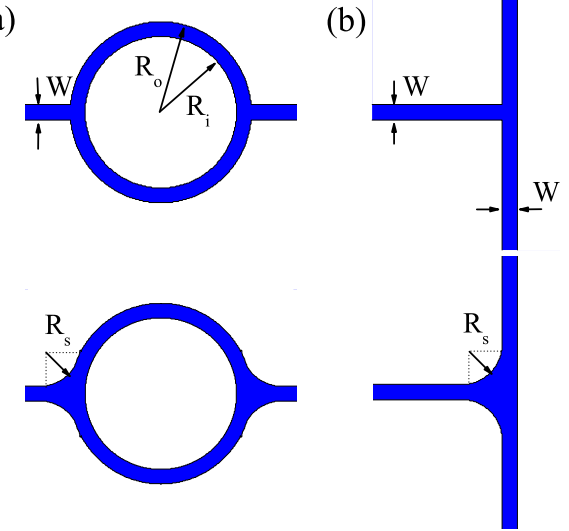

FIG. 1. (Color online) Potential profiles for (a) rings and (b) T wires, considering (top) right-angle and (bottom) smooth connections. The smooth connections are described by circles of radius $R_{s}=300 \AA$. The width in both systems is $W=100 \AA$ and the average radius is $R_{\mathrm{av}}=600 \AA$. The potential is defined as $V(x, y)=0$ inside ring and channel regions (blue) and $V_{e}$ outside (white).

better control on how many subbands are accessible for the wave packets.

Our theoretical approach follows the one of Refs. 9 and 10 , where we inject a Gaussian wave packet into the ring from the left lead in a particular subband and calculate numerically "exact" (i.e., the results can be calculated for a given accuracy) its time evolution by solving the timedependent Schrödinger equation. This approach allows us to find the transmission and reflection coefficient and also possible bound states in the junctions.

The paper is organized as follows: in Sec. II, we present our theoretical model and discuss the numerical technique to solve the time-dependent Schrödinger equation. Symmetric rings are discussed in Sec. III. The influence of asymmetry between the upper and lower arms of the ring on the $\mathrm{AB}$ effect is studied in Sec. IV by inserting a Gaussian potential barrier or an impurity in the ring arm. Our conclusions are presented in Sec. V. The bound and resonant states in the lead-arm junction are investigated in the Appendix.

\section{THEORETICAL MODEL}

Our system consists of a planar quantum ring and channels for electron injection so that the motion of the electrons is constrained to be in the $(x, y)$ plane (see Fig. 1). The electrons are considered within the effective-mass approximation and they are confined by a step potential, i.e., $V(x, y)=0$ inside the quantum ring and leads, and $V(x, y)=V_{e}$ otherwise, where $V_{e}$ is the conduction-band offset. In order to understand the dynamics of a wave packet passing through a channel-ring connection, we made also a brief study of a T-shaped wire.

Figure 1 shows a schematic view of our (a) rings and (b) $\mathrm{T}$ wires. The quantum ring is defined by two concentric circles of radii $R_{i}=550 \AA$ and $R_{o}=650 \AA$ so that the average radius of the system is $R_{\mathrm{av}}=\left(R_{i}+R_{o}\right) / 2=600 \AA$ and the width is $W=100 \AA$. The same width $W$ is considered for the left and right leads. For $\mathrm{T}$ wires, all channels have the same width $W$. Two different lead-ring connections will be studied: (1) a right-angle connection and (2) a smooth (adiabatic) connection. The smooth connections are described by circles of radius $R_{s}$, which are tangent to the walls of the channels and to the external circle of the ring. In the examples of Fig. 1 (bottom), the circles describing the smooth connections have radius $R_{\mathrm{s}}=300 \AA$.

An electron confined in a quasi-one-dimensional channel of width $W$ has subband energy

$$
E_{n}\left(k_{x}\right)=E_{n}^{(y)}+\frac{\hbar^{2} k_{x}^{2}}{2 m_{e}},
$$

where $E_{n}^{(y)}=n^{2} \pi^{2} \hbar^{2} / 2 m_{e} W^{2}$, if $V_{e}$ is very large (for our numerical results we calculated $E_{n}^{(y)}$ numerically for finite $V_{e}$ value). In our approach, we inject a Gaussian wave packet, propagating in the $x$ direction, from left to right,

$$
\psi(x, y)=\frac{1}{\sigma \sqrt{2 \pi}} \exp \left[-\frac{\left(x-x_{0}\right)^{2}}{2 \sigma^{2}}+i k_{x}^{i} x\right] \phi_{n}(y),
$$

where $\phi_{n}(y)$ is the wave function of the $n$th eigenstate of the quantum well in the $y$ direction, and $k_{x}^{i}=\sqrt{2 m_{e} \varepsilon_{i} / \hbar^{2}}$, with $m_{e}$ and $\varepsilon_{i}$ as the electron effective mass and kinetic energy, respectively. The parameter $\sigma$ gives the width of the wave packet, in the $x$ direction.

The time evolution of the wave packet is studied by means of the split-operator technique, which consists in separating kinetic and potential terms in the time-evolution operator,

$$
\begin{aligned}
\Psi(\mathbf{r}, t+\Delta t)= & \exp \left[-\frac{i H \Delta t}{\hbar}\right] \Psi(\mathbf{r}, t) \\
= & e^{-i V \Delta t / 2 \hbar} e^{-i T_{x} \Delta t / \hbar} e^{-i T_{y} \Delta t / \hbar} e^{-i V \Delta t / 2 \hbar} \Psi(\mathbf{r}, t) \\
& +O\left(\Delta t^{3}\right),
\end{aligned}
$$

where $T_{x(y)}$ is the kinetic-energy operator for $x(y)$ direction and the error $O\left(\Delta t^{3}\right)$ is a consequence of the noncommutativity of kinetic and potential terms. ${ }^{11}$ If a magnetic field is present, an additional $O\left(\Delta t^{2}\right)$ error appears in this expression because of the position-dependent vector potential in the kinetic terms. The potential terms are directly multiplied by the functions whereas the kinetic terms are rewritten in the Cayley form ${ }^{12}$ and a finite difference scheme is used to calculate the derivatives. ${ }^{13-15}$ The main advantage of this technique lies in the separation of the kinetic terms for each direction; this separation allows one to study a system of many degrees of freedom without dealing with gigantic matrices; instead, only a set of small matrices, one for each direction, appears in this approach.

Special care has to be taken for the boundary conditions at the entrance and exit of the leads. The main problem is that, as the wave packet reaches the edges of the computational box, it undergoes a spurious reflection, when one imposes that the wave function is zero at the boundaries. One possible way to overcome this problem is to consider a very long channel so that the wave packet would take a long time to reach the edge of the system, as was done in Refs. 9 and 10. However, this would lead to large computational costs, 
hence, alternative ways to deal with this problem have been developed. Kosloff and Kosloff ${ }^{16}$ suggested the use of absorbing (imaginary) potentials at the edges, in addition to the real confinement potential of the system. Such an imaginary potential gradually absorbs the wave function before it reaches the edges, avoiding spurious reflection effects. Several forms of imaginary potential has been suggested and analyzed. ${ }^{17,18}$ Another interesting way to solve this problem was suggested by Arnold et al. ${ }^{19}$ where completely transparent discrete boundary conditions were developed, based on the Laplace method for solving the time-dependent Schrödinger equation. However, the method was developed only for one-dimensional problems, using the Cayley form and the Crank-Nicolson scheme. A generalization of this method to two dimensions within the split-operator treatment would be needed in our case. Therefore, in this paper we considered the imaginary potential suggested by Manolopoulos, ${ }^{20}$ which can be tuned to be free of reflections on the edge of the system and also at the beginning of the imaginary potential region, for a wide range of initial energies. This imaginary potential, which depends only on the direction of propagation $x$, is given by

$$
V_{\mathrm{im}}(\bar{x})=-i E_{\min }\left[a \bar{x}-b \bar{x}^{3}+\frac{4}{(c-\bar{x})^{2}}-\frac{4}{(c+\bar{x})^{2}}\right],
$$

where $a=1-16 / c^{3}, b=\left(1-17 / c^{3}\right) / c^{2}, c=2.62206$ and $E_{\min }$ is the lowest electron energy allowed to be considered, which can be calculated from Eq. (2.27) of Ref. 20 as

$$
E_{\min }=\frac{\hbar^{2}}{2 m_{e}}\left[\frac{c}{2\left(x_{2}-x_{1}\right) \delta}\right]^{2}
$$

for an imaginary potential localized between $x_{1}$ and $x_{2}$. The accuracy parameter $\delta$ is chosen as 0.2 , to minimize reflections at $x_{1}$. The variable $\bar{x}$ depends on $x$ as

$$
\bar{x}=2 k_{\min } \delta /\left(x-x_{1}\right),
$$

where $k_{\min }=\sqrt{2 m_{e} E_{\min } / \hbar^{2}}$. With these expressions, we choose $x_{2}$ as the edge of the system and $x_{1}=420 \AA$ before the edge, leading to a minimum energy $E_{\min } \sim 25 \mathrm{meV}$ for $m_{e} / m_{0}=0.041$.

The transmission $T$ and reflection $R$ probabilities are calculated by integrating the component of the probability current in the direction of propagation, at some fixed points $x_{R}$ and $x_{L}$, localized in the right and left channels, respectively,

$$
T=\int_{0}^{\infty} d t \int_{-\infty}^{+\infty} d y J_{x}\left(x_{R}, y, t\right)
$$

and

$$
R=-\int_{0}^{\infty} d t \int_{-\infty}^{+\infty} d y J_{x}\left(x_{L}, y, t\right)
$$

where the $x$ component of the probability current is defined as

$$
J_{x}(x, y, t)=-i \frac{\hbar}{2 m_{e}}\left(\Psi^{*} \frac{\partial}{\partial x} \Psi-\Psi \frac{\partial}{\partial x} \Psi^{*}\right)+\frac{e}{m_{e}} A_{x} \Psi^{*} \Psi
$$

The time integrals in Eqs. (7) and (8) are performed numerically up to a large value of time, in order to let the wave packet pass entirely through the point where $J_{x}$ is measured. We checked carefully that the sum of transmission and reflection probabilities is $T+R=1$ with an accuracy of at least $0.1 \%$. The wave packets passing through the points $x_{R}$ and $x_{L}$ travel in opposite directions, hence, a minus sign is put in the expression for $R$ [Eq. (8)]. The magnetic field is always considered perpendicular to the plane of motion, i.e., in the $z$ direction and the vector potential is taken in the Coulomb gauge $\mathbf{A}=(-y, x, 0) B / 2$.

In order to study the scattering of the electron into different subbands, we project the wave function on the $j$ th eigenstate of the quantum well at a fixed point $x_{i}$,

$$
P_{j}\left(x_{i}, t\right)=\left|\left\langle\Psi \mid \phi_{j}\right\rangle\right|^{2}=\left|\int_{-\infty}^{+\infty} d y \Psi\left(x_{i}, y, t\right) \phi_{j}(y)\right|^{2} .
$$

This is the probability to find the electron in the $j$ th subband at position $x_{i}$ per length in $x$ direction. The contribution of each subband state to the probability current can be calculated by

$$
J_{x}^{(j)}(x, t)=-i \frac{\hbar}{2 m_{e}}\left(\bar{P}_{j}^{*} \frac{\partial}{\partial x} \bar{P}_{j}-\bar{P}_{j} \frac{\partial}{\partial x} \bar{P}_{j}^{*}\right),
$$

where the function $\bar{P}_{j}(x, t)=\left\langle\phi_{j} \mid \Psi\right\rangle$ gives the part of the time-dependent wave function which is in the $j$ th subband. The quantity

$$
\left\langle J_{x}^{(j)}\right\rangle=\int_{0}^{\infty} d t J_{x}^{(j)}\left(x_{r}, t\right)
$$

is the total (or time averaged) current in the $j$ th subband passing through the cross section of the channel at $x=x_{r}$. Notice that, since the function $\bar{P}_{j}(x, t)$ is not normalized, $\left\langle J_{x}^{(j)}\right\rangle$ is not a probability and therefore, its value can be larger than one. Finally, the time-dependent probability current at a point $x_{i}$ is given by

$$
J_{T}\left(x_{i}, t\right)=\int_{-\infty}^{+\infty} J_{x}\left(x_{i}, y, t\right) d y
$$

and will help to understand the trajectory of the wave packet through the channels. Notice, however, that the timedependent probability current $J_{T}$ defined in Eq. (12) measures only the propagation of a pulse in a system in the absence of a bias between the leads, hence it is not related to a steady-state current, but rather to a transient current, which is different from the currents defined in previous papers, ${ }^{21}$ which were obtained by means of Green's functions.

With the present approach, we are also able to find bound states, by propagating an arbitrary initial wave function in the imaginary time domain. ${ }^{13}$ Since any wave function can be written as a linear combination of eigenstates 


$$
|\Psi\rangle_{t}=\sum_{n=0}^{\infty} a_{n} e^{-i E_{n} t / \hbar}\left|\Phi_{n}\right\rangle,
$$

where $\Phi_{n}$ and $E_{n}$ are, respectively, the eigenfunctions and eigenenergies of the $n$th eigenstate. If one takes $\tau=i t$, with $\tau \rightarrow \infty$, the term corresponding to the ground state will dominate over the others and the function must converge to the ground state of the system as $\tau$ increases. Excited states can also be obtained by using Gram-Schmidt orthonormalization by choosing as an initial wave-function one that is orthonormal to the ground state. Consequently, the ground-state wave function will be absent in the sum of Eq. (13), thus, the lowest-energy term in the sum is $E_{1}$ and hence, the wave function will converge to $\left|\Phi_{1}\right\rangle$ as $\tau \rightarrow \infty$. To obtain $\Phi_{2}$, one starts with a function which is orthogonal to $\Phi_{1}$ and $\Phi_{0}$, and so on.

\section{SYMMETRIC RING}

We used our model to study the quantum confinement and the time evolution of a wave packet as described in the previous section, with $x_{0}=-1100 \AA, \sigma=200 \AA$, and $n=1$ (ground state for transverse motion) or 2 (first-excited state) [see Eq. (2)], inside a InGaAs/InAlAs heterostructures, where the conduction-band effective mass and band offset are taken as $m_{e}=0.041 m_{0}$ and $V_{e}=600 \mathrm{meV}$, respectively. For a channel with width $W=100 \AA$, this leads to subband energies $E_{n}^{(y)} \simeq 53,207$, and $443 \mathrm{meV}$, for $n=1,2$, and 3, respectively. The corresponding values for $V_{e} \rightarrow \infty$ are 91.7, 366.8 , and $825.3 \mathrm{meV}$. Notice that the results for finite $V_{e}$ satisfies approximately $E_{2}^{(y)} \sim 4 E_{1}^{(y)}$ and $E_{3}^{(y)} \sim 9 E_{1}^{(y)}$.

\section{A. Geometry-induced localized states}

It is well known that a right-angle connection in $\mathrm{T}$ wires leads to one, and only one, confined state. ${ }^{13,22}$ At the crossing point, the confined state has a zero-point energy lower than the ground-state energy of the quantum wells formed in the leads, which then act like barrier potentials around the region of the junction (see the Appendix for more details). Since the connections between the leads and the ring are similar to T-wire junctions, it is expected that two (degenerate), and only two, confined states would be observed in the channelring system, since, in this case, two connections are involved, leading to symmetric and antisymmetric states. This is observed, indeed, for simple channel-ring connections, as shown in Fig. 2(a). No confined excited state is observed, besides the practically degenerate antisymmetric state. The binding energy of this degenerate state is found to be $E_{0}$ $\simeq 46 \mathrm{meV}$, which is $7 \mathrm{meV}$ lower than the ground-state energy of the quantum well mentioned earlier, $E_{0}^{(y)} \simeq 53 \mathrm{meV}$.

However, real systems have smooth connections, which can result in several nondegenerate bound states. We found that in this case, it is possible to have excited bound states that are localized at the lead-arm crossing region, as illustrated in Figs. 2(b) and 2(c), where the wave functions for the ground and second-excited states are shown, respectively, for a smooth connection with $R_{s}=300 \AA$.

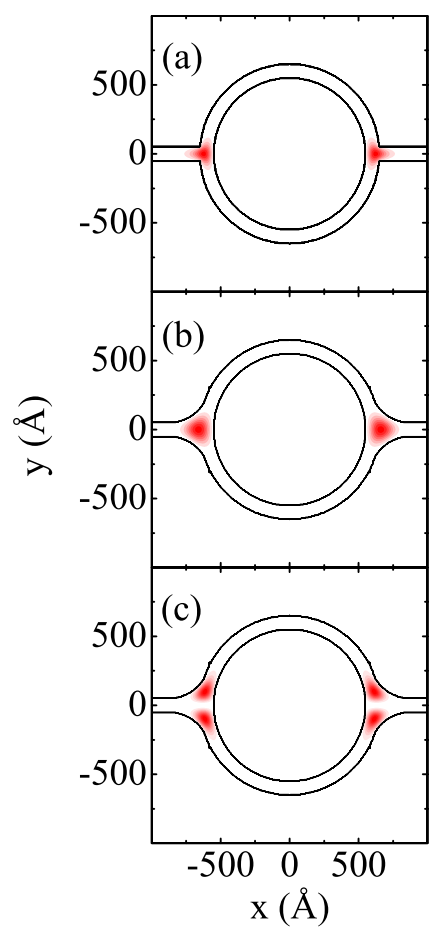

FIG. 2. (Color online) (a) Ground-state squared wave function (ground and first-excited states are degenerate states with symmetric and antisymmetric wave functions), for sharp channel-ring connections. (b) Ground and (c) second-excited-states squared wave functions, for smooth connections. Black lines illustrate the limits of the channel-ring profiles.

\section{B. Time evolution for $B=0$}

Three wave packets were considered, with average kinetic energies $\varepsilon_{1}=70 \mathrm{meV}, \varepsilon_{2}=120 \mathrm{meV}$, and $\varepsilon_{3}=180 \mathrm{meV}$, and $\phi_{n=1}(y)$, i.e., localized in the lowest subband. The corresponding average wave vector $k_{x}^{i}$ is qualitatively illustrated in Fig. 3, where the parabolic energy subbands of the quantum well of width $W$ created by the channels and ring arms are also shown. For $\varepsilon_{3}$, we have deliberately chosen a value larger than the energy of the second subband of the quantum well, which is about $153 \mathrm{meV}$ above the first subband. This will allow us to observe the influence of this subband on the wave packet and on the physical properties of the system as well. As illustrated in Fig. 3, two values of $k_{x}$, defined as $k_{3}^{(1)}$ and $k_{3}^{(2)}$, are possible for $\varepsilon_{3}$, one for each subband. Our results for $\varepsilon_{3}$ are for $k_{3}^{(1)}$ by default, except when explicitly mentioned that we are dealing with $k_{3}^{(2)}$. Since we are considering a wave packet, the initial wave function does not contain a single $k_{x}$ value but rather a distribution of $k_{x}$ 's around $k_{x}^{(i)}$, with width $\Delta k_{x}$, as illustrated in the right part of Fig. 3. This variation in $k_{x}$ yields an energy distribution $\Delta E$, as illustrated in Fig. 3. For an initial wave packet as given by Eq. (2), the width in $k_{x}$ space can be easily obtained from a Fourier transform of the wave function

$$
\bar{\psi}\left(k_{x}, y\right)=\frac{1}{2 \pi} e^{-i\left(k_{x}-k_{x}^{(i)}\right) x_{0}} e^{-\left(k_{x}-k_{x}^{(i)}\right)^{2} \sigma^{2} / 2} \phi_{n}(y) .
$$

It is easily seen that in reciprocal space the squared wave function is also a Gaussian but with a width proportional to 


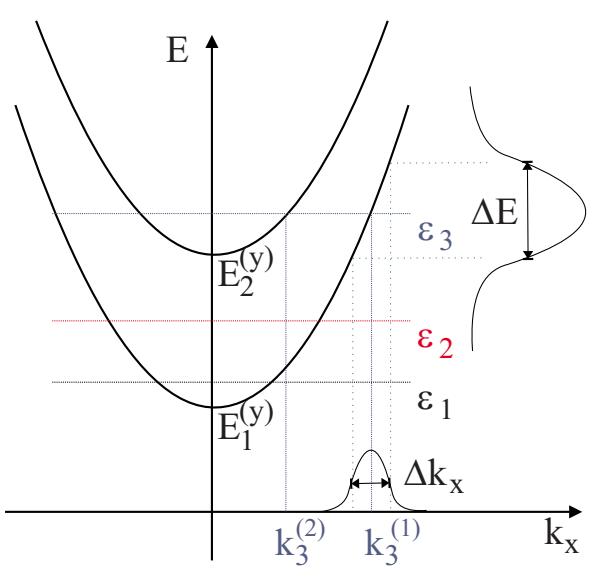

FIG. 3. (Color online) Energy versus wave-vector $k_{x}$ diagram for a quantum wire (solid curves). The average energies of the considered three wave packets, $\varepsilon_{1}, \varepsilon_{2}$, and $\varepsilon_{3}$ (thick dotted lines) are indicated. The widths of $k_{x}$ and $E$ distributions of the initial wave packet $\varepsilon_{3}$ are represented by horizontal and vertical Gaussians, respectively. The wave vector $k_{3}^{(1)}$ is related to the energy $\varepsilon_{3}$ of the ground-state subband whereas $k_{3}^{(2)}$ is for the same energy but for the first-excited subband.

$1 / \sigma$. For the parameters chosen in the present work, we calculate from the full width at half maximum $\Delta x=2 \sqrt{2 \ln 2} \sigma$ $\sim 470.96 \AA$ and $\Delta k_{x}=2 \sqrt{2 \ln 2} / \sigma \sim 0.011774 \AA^{-1}$. With the later, we can calculate the width $\Delta E$ of the energy distribution of the wave packet

$$
\frac{\hbar^{2}\left(k_{x}^{i}+\Delta k_{x}\right)^{2}}{2 m_{e}}=\frac{\hbar^{2} k_{x}^{i 2}}{2 m_{e}}+\frac{\hbar^{2} k_{x}^{i} \Delta k_{x}}{m_{e}}+\frac{\hbar^{2} \Delta k_{x}^{2}}{2 m_{e}},
$$

where we identify $\hbar^{2} k_{x}^{i 2} / 2 m_{e}$ as the energy $\varepsilon_{i}$ and

$$
\Delta E=\frac{\hbar^{2} k_{x}^{i} \Delta k_{x}}{m_{e}}+\frac{\hbar^{2} \Delta k_{x}^{2}}{2 m_{e}} .
$$

From this expression and the value for $\Delta k_{x}$ mentioned previously, we obtain $\Delta E \sim 72,90$, and $108 \mathrm{meV}$, for $\varepsilon_{1}, \varepsilon_{2}$, and $\varepsilon_{3}$, respectively.

Solving the time-dependent Schrödinger equation gives us $\Psi(x, y, t)$. The projections of the time-dependent wave function on the ground (black, $P_{1}$ ) and first-excited (red, $P_{2}$ ) subband states of the channels were calculated numerically as functions of time at three different points of the system: $x_{l}=-1100 \AA$ (left channel), $x_{a}=0 \AA$, (upper arm) and $x_{r}$ $=1100 \AA$ (right channel). The results are depicted in Figs. 4(a)-4(c), for $\varepsilon_{1}, \varepsilon_{2}$, and $\varepsilon_{3}$, respectively. A few remarkable facts are observed: (1) the electron is reflected and transmitted through the channels in packages, which are a consequence of the reflections at the lead-ring intersection points. (2) The wave function in the left and right channels is restricted to the lowest subband and thus has zero projection over the first-excited state. Remarkably, inside the ring, the wave function is partially scattered to the second subband. For $\varepsilon_{3}$, the projection on the second subband can even be dominant and larger than the projection on the ground state. Although $\varepsilon_{2}$ is below the second subband, there is still a small nonzero projection $P_{2}$ on the second subband, due to
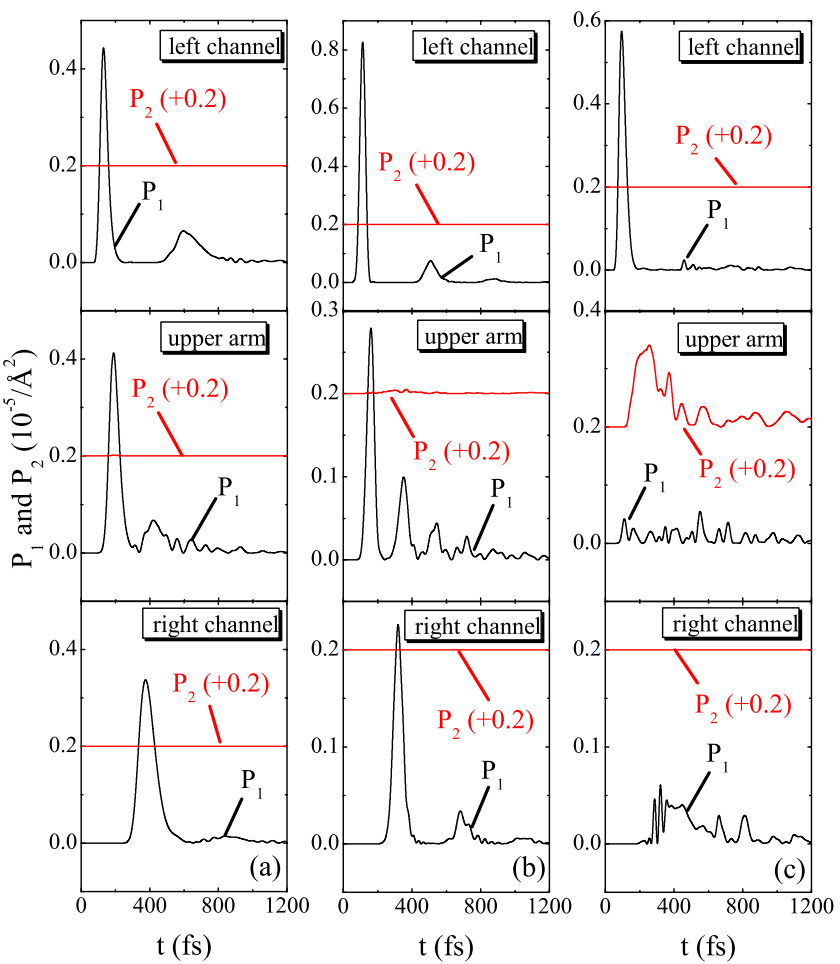

FIG. 4. (Color online) Time evolution of the projection of the wave function on the ground (black) and first-excited (red) subbands of a quantum well of width $W=100 \AA$, calculated at three different locations: left channel, upper arm of the ring, and right channel. The injected wave packet is in the lowest subband and has average energy (a) $\varepsilon_{1}$, (b) $\varepsilon_{2}$, and (c) $\varepsilon_{3}$. [see Fig. 3]

the wide distribution of $k_{x}$ 's of the initial wave functions. Thus, the electron wave packet partly scatters to the second subband at the first lead-arm connection but it scatters back to the lowest subband at the second lead-arm connection, leading to an outgoing wave function that fully resides in the lowest subband. In Fig. 5, we consider a wave packet with average energy $\varepsilon_{3}$ that is centered around $k_{3}^{(2)}$, in the second subband, so that the projection $P_{1}$ is zero at both leads. Again, the projections $P_{1}$ and $P_{2}$ are nonzero inside the quantum ring arm. Although not shown in the figures, we have also calculated the projections over the second-excited state $P_{3}$ and we found zero for the three cases of energy, inside the leads and the ring arms. However, as depicted in Fig. 6, considering a wave packet in the second subband with energy $430 \mathrm{meV}$, which is above the edge of the third subband of the system, which is about $390 \mathrm{meV}$, the projection $P_{3}$ (blue) is nonzero inside the quantum ring arm. Notice that the peaks of $P_{2}$ and $P_{3}$ are shifted to larger time as compared to the peaks in $P_{1}$. This time delay is a consequence of the fact that $k_{x}^{(1)}>k_{x}^{(2)}>k_{x}^{(3)}$ so that the wave packets in higher subbands move with lower velocity. These results clearly show that, owing to the fact that we are dealing with a finite width system, the wave function is not only allowed to reflect at the walls of the confinement potential of the ring and channels but it is also allowed to access other subbands, when it is inside the ring, as a consequence of scattering at the arm-ring connections. At the connection between the ingoing lead and the ring the wave packet can scatter into other 


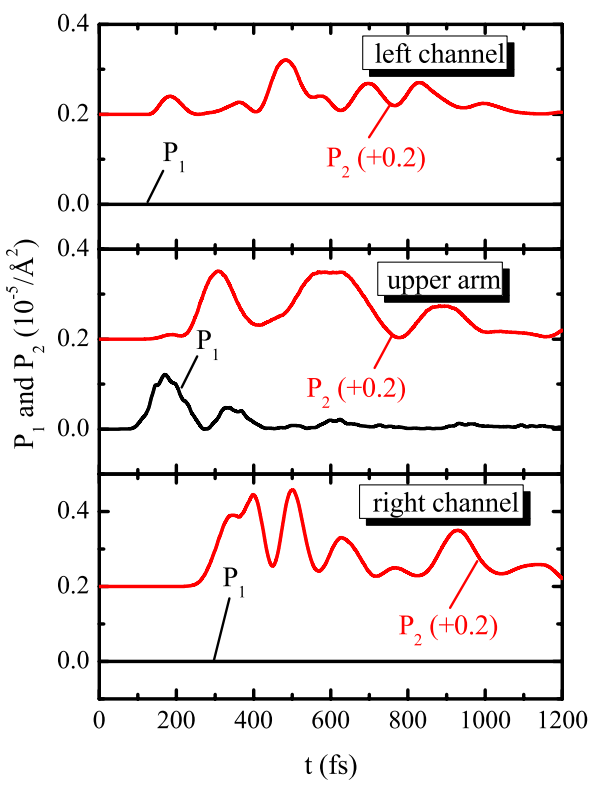

FIG. 5. (Color online) The same as Fig. 4(c) but now we consider the wave packet with energy $\varepsilon_{3}$ to be in the second subband, with wave vector $k_{3}^{(2)}$.

subbands if energy conservation is satisfied. When part of the wave is scattered to the other subbands, a difference in phase of $\pi$ is picked up between the left- and right-going waves (as viewed from the direction of the incoming wave) that leads to a destructive interference at the outgoing lead.

Both characteristics can also be illustrated by the time evolution of the contour plots of the squared wave functions. They are shown in Figs. 7(a) and 7(b), for four different time steps, starting with wave packets of energy $\varepsilon_{1}$ and $\varepsilon_{2}$, respectively. Figures 8(a) and 8(b) are for $\varepsilon_{3}$ in subbands $k_{3}^{(1)}$ and $k_{3}^{(2)}$, respectively. Right-angle (left panels) and smooth (right panels) lead-ring connections were considered. For the smooth case, the wave function exhibits a complicated interference pattern at the connections.

The presence of the packages observed in Fig. 4, due to the reflections at the lead-ring intersection points, can be

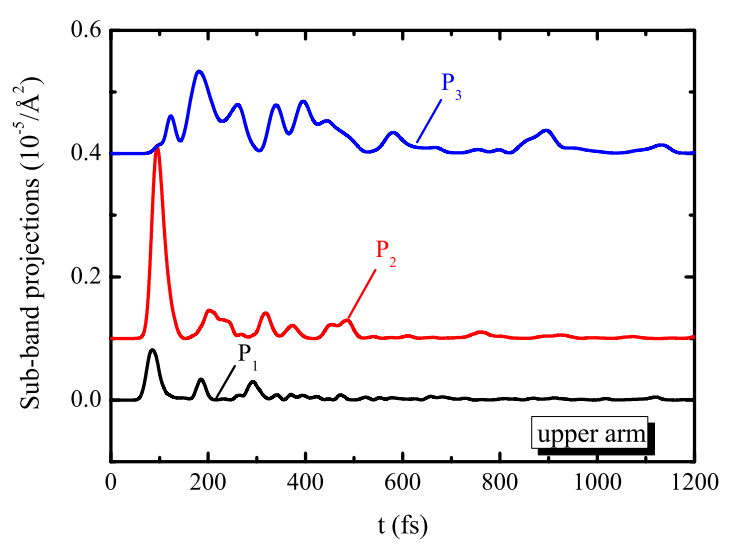

FIG. 6. (Color online) Time evolution of the wave-function projections on the ground (black), first-excited (red), and secondexcited (blue) subband states of a quantum well of width $W$ $=100 \AA$, calculated at the upper arm of the ring, for a wave packet in the second subband with energy $430 \mathrm{meV}$. (a)

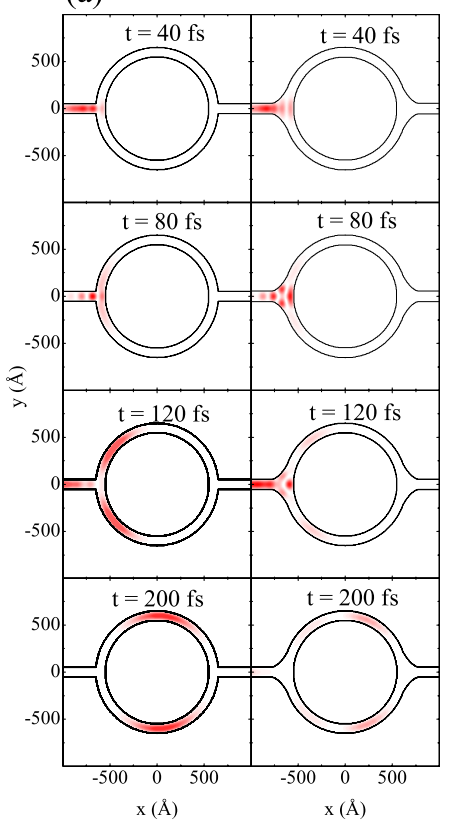

(b)

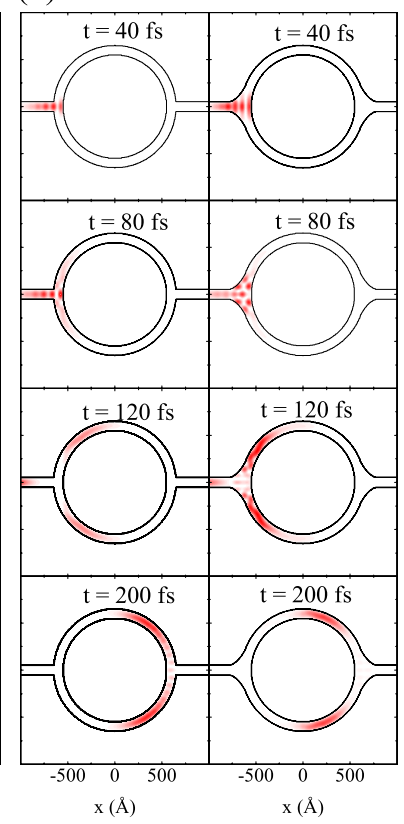

FIG. 7. (Color online) Contour plots of the time evolution of the squared wave function, for right-angle (left panels) and smooth (right panels) lead-ring connections. The initial wave packet is in the lowest subband with width $\sigma=200 \AA$ and energy (a) $\varepsilon_{1}$ and (b) $\varepsilon_{2}$.

better understood if one looks at the time-dependent probability current in Fig. 9, which is calculated over the same three points, $x_{l}, x_{a}$, and $x_{r}$, where the wave-function projections were calculated previously, for $\varepsilon_{1}$ (black, solid), $\varepsilon_{2}$ (red, dashed), and $\varepsilon_{3}$ (blue, dotted). It is easily seen that there is more than one peak in the probability current in left and right leads. At the left channel, the positive peak is due to the incoming packet and the negative peaks are the reflected waves, which are separated by a region of zero probability current. This can also be understood from the time snapshots of the electron density shown in Figs. 7 and 8. In the upper arm of the ring, the probability current has both positive and negative peaks, meaning that the wave function traveled back and forth inside the ring arms. At the right lead, more than one positive peak is observed. This effect can be understood if one analyzes the trajectory of the wave packets through the system; the parts of the wave function which come from the first junction and travel through the upper and lower arms of the ring interfere at the second junction, and from this interference, part of the wave function is transmitted to the right channel, giving a contribution to the transmission probability while the other part travels back through the arms to the first junction (see Figs. 7 and 8). Back at the first junction, these waves interfere again, resulting in a wave that is transmitted into the left channel, hence, contributing to the reflection probability, and another pair of waves travels through the upper and lower arms of the ring toward the second junction again, and so on.

We estimate the times for a classical particle to perform these trajectories and found good agreement with our quantum simulations. For $\varepsilon_{1}$ (black-solid lines) in Fig. 9, the first negative peak in the left channel and the first positive peak in 
(a)

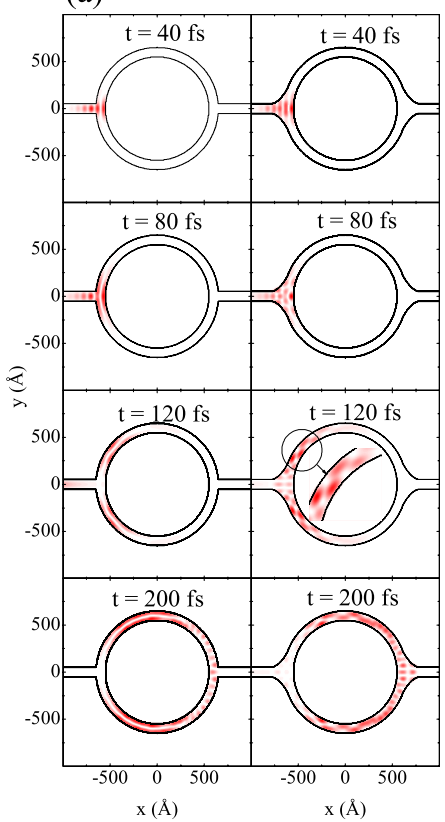

(b)

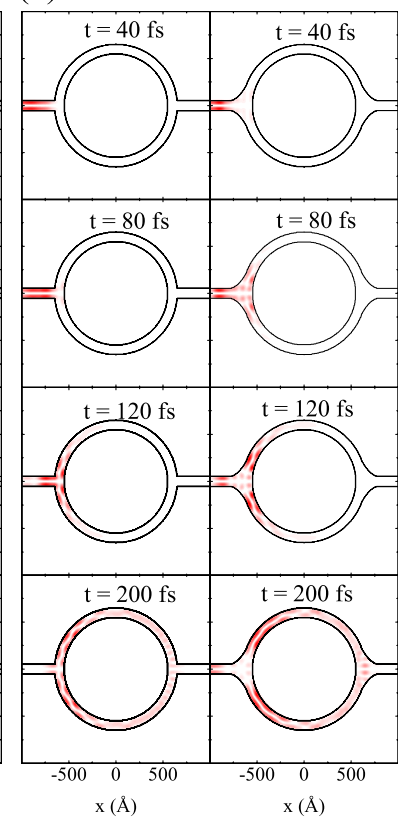

FIG. 8. (Color online) Contour plots of the time evolution of the squared wave function, for right-angle (left panels) and smooth (right panels) lead-ring connections. The initial wave packet has width $\sigma=200 \AA$, energy $\varepsilon_{3}$, and is in the (a) lowest subband with wave vector $k_{3}^{(1)}$, and in the (b) second subband with wave vector $k_{3}^{(2)}$.

the right channel are found at 128.8 and 371.8 fs whereas for a classical particle, the reflected and transmitted times are 129.0 and $372.3 \mathrm{fs}$, respectively. The first positive peak in the upper arm of the ring is observed at $186.1 \mathrm{fs}$, which agrees with the time for a classical electron to reach this point,

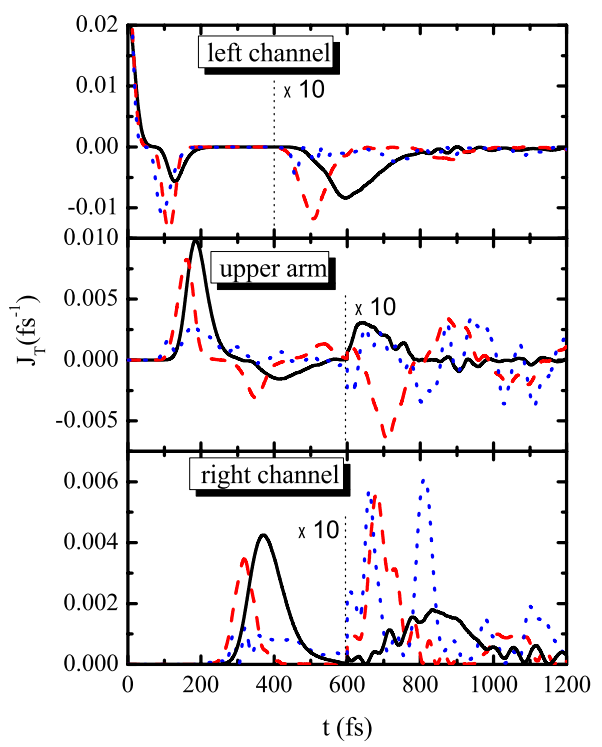

FIG. 9. (Color online) Time-dependent probability current, calculated at three points: left channel, upper arm of the ring, and right channel, for wave packets with energies $\varepsilon_{1}$ (black, solid), $\varepsilon_{2}$ (red, dashed), and $\varepsilon_{3}$ (blue, dotted) in the case of sharp lead-ring connections.

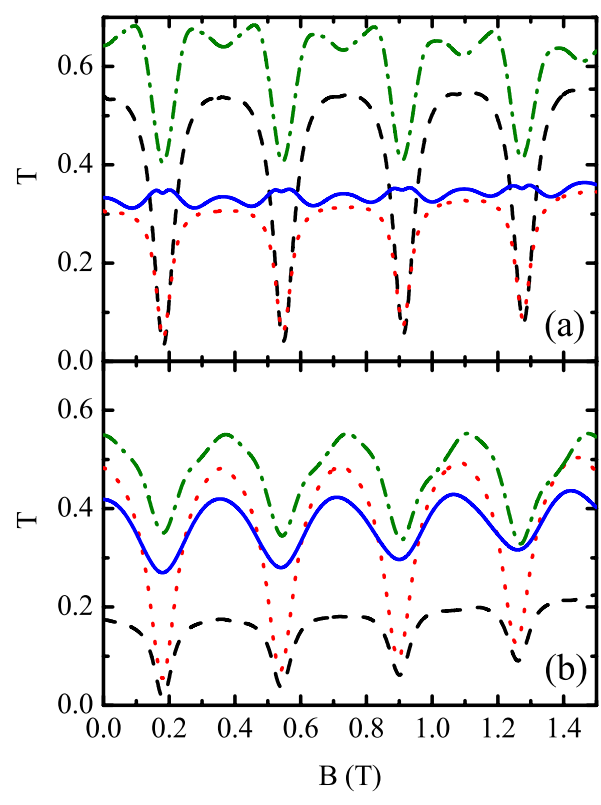

FIG. 10. (Color online) Transmission probabilities $T$ for wave packets with energies $\varepsilon_{1}$ (black, dashed) and $\varepsilon_{2}$ (red, dotted) in the first subband, and with energy $\varepsilon_{3}$ in the first (blue, solid) and second (green, dashed-dotted) subbands, as functions of the magnetic field, for (a) right-angle and (b) smooth lead-ring connections.

estimated as 186.2 fs. For the second reflected and transmitted packets, i.e., second negative (positive) peak in left (right) channel, which occur, respectively, at 594.8 and 832.2 fs, the times for a classical particle are slightly larger, 615.5 fs for reflection and 858.7 fs for transmission. Besides, the time for the second (negative) peak in the upper arm is 413.0 fs while for a classical particle, this time is estimated as 429.4 fs. Contrarily, considering a wave packet with energy $\varepsilon_{2}$, the times for reflected (transmitted) classical particles are found as 98.6 (284.3) fs, which are lower than the values found for the first negative (positive) peak in left (right) channel, which occurs at 112.4 (318.0) fs. The upper arm first peak is at $161.0 \mathrm{fs}$ while for a classical particle, this time is also lower, $142.2 \mathrm{fs}$.

The snapshots shown in Figs. 7 and 8 illustrate clearly: (i) wave interference at the junctions, (ii) the transmission of the injection lead into the arms of the ring and the backscattering into the leads, and (iii) the radial distribution of the electron in the ring arm, i.e., with a maximum in the center of the channel for an electron wave packet in the lowest subband or a two-peak radial structure in case of an electron propagating in the second subband [see, e.g., $t=120$ fs pictures in Figs. 7(b), 8(a), and 8(b)]. In the latter case, the maxima of the wave function traces the classical path of an electron bouncing off the inner and outer ring boundaries.

\section{Time evolution for nonzero $B$}

The interference of the electron wave packet can be influenced by threading a magnetic flux through the ring. Figure 10 shows the calculated transmission probability $T$ as a function of the applied magnetic field, for the previous three values of the wave packet energy. The well-known Aharonov- 
Bohm periodic oscillations in $T$ are clearly seen and the amplitude of such oscillations decreases as the magnetic field increases, an effect that is due to the imbalance of injection into the upper and lower arms of the ring as a consequence of the Lorentz force. ${ }^{9}$

Comparing the results for right-angle and smooth leadring connections in Figs. 10(a) and 10(b), respectively, we notice that, depending on the energy of the wave packet, the transmission probability and the amplitude of the AB oscillations can be either increased or reduced by the smoothness of the connection. This is surprising because one would intuitively expect that a smooth connection should always increase the transmission probability since the effective area in the junction is larger for the smooth case. However, one must remember that the lead-ring junction acts like a confining potential, as discussed in Sec. III A, where quantum resonance effects are present for some electron energies, and the existence of a smooth junction changes this resonances spectrum, which suggests that both the wave packet energy and the smoothness of the junction must play an important role in the transmission probability. This effect is discussed in more details in the Appendix, for a T-wire junction.

For $\varepsilon_{3}$ in the first subband, the oscillations in the transmission probability have a very small amplitude and the average transmission in both cases of sharp and smooth junctions are practically the same. In the case of a sharp junction, the amplitude of the $\mathrm{AB}$ oscillations is very weak and more minima are observed as compared to the results for $\varepsilon_{1}$ and $\varepsilon_{2}$. The fact that in this case the transmission probability is far from 0 at $\Phi=(n+1 / 2) \phi_{0}$, where $\phi_{0}$ is the flux quantum (although it shows a small minimum), as one would expect for an ideal QR, suggests that the interference that occurs in ideal rings, which is responsible for $T=0$ at these points, is not completely destructive in our system, specially for $\varepsilon_{3}$. Thus, for these values of $\Phi$, part of the wave packet undergoes destructive interference, which reduces the transmission probability and results in a small minimum but the other part continues and circles again over the ring so that a new interference occurs at the other junction, resulting in a doubling of the $\mathrm{AB}$ frequency or equivalently minima for $\Phi$ $=(n+1 / 2) \phi_{0} / 2$. This behavior can also be observed in Fig. 11(a), where the transmission probability $T$ is shown as a function of the magnetic field for three values of wave packet energy, $\varepsilon=140$ (black, dashed), 160 (red, dotted), and 170 (blue, solid) meV. The latter two energy values are higher than the second subband energy. For $\varepsilon=140 \mathrm{meV}, T$ exhibits the common $\mathrm{AB}$ oscillations, but for the other energies, the oscillation can be described by a superposition of two patterns of oscillations, one with minima at $\Phi=(n+1 / 2) \phi_{0}$ and the other at $\Phi=(n+1 / 2) \phi_{0} / 2$ in case of sharp lead-ring connection. For smooth lead-ring connections, this effect is not observed, as shown in Fig. 11(b) because of a stronger interference effect at the junctions.

If a $B=0.18 \mathrm{~T}$ magnetic field is applied perpendicular to the ring plane, as shown in Fig. 12(a) for $k_{3}^{(1)}$, the outgoing wave function in the right lead exhibits two peaks. From this result, one can infer that the outgoing wave function has a large projection $P_{2}$ in the right channel, i.e., it can occupy the second subband in this channel. Conversely, if we inject a wave packet in the second subband with wave vector $k_{3}^{(2)}$, as

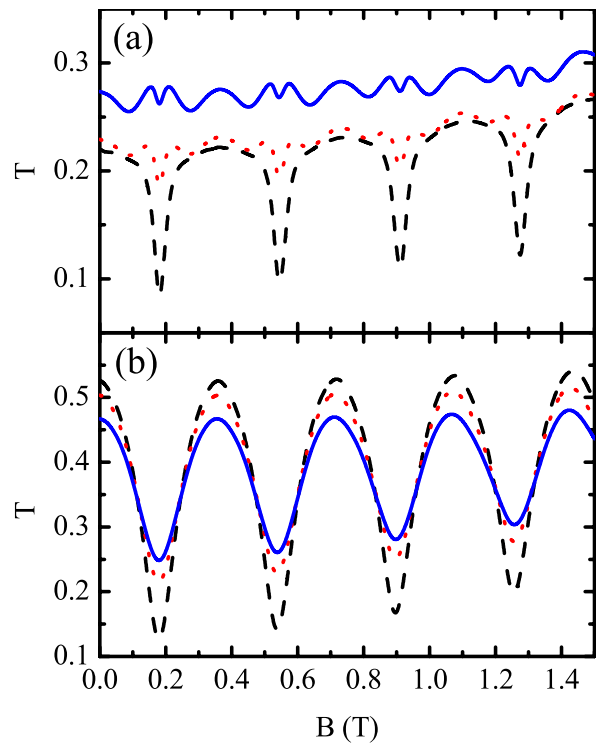

FIG. 11. (Color online) Transmission probabilities $T$ for wave packets injected in the lowest subband with different energies $\varepsilon$ = 140 (black, dashed), 160 (red, dotted), and $170 \mathrm{meV}$ (blue, solid), as functions of the magnetic field, considering (a) right-angle and (b) smooth with $R_{s}=300 \AA$ lead-ring connections.

shown in Fig. 12(b), the outgoing wave function has a maximum in the center of the lead, which suggests that the projection $P_{1}$ is large in the right lead for this value of magnetic field. This is an effect that was absent when $B=0$. For non- (a)

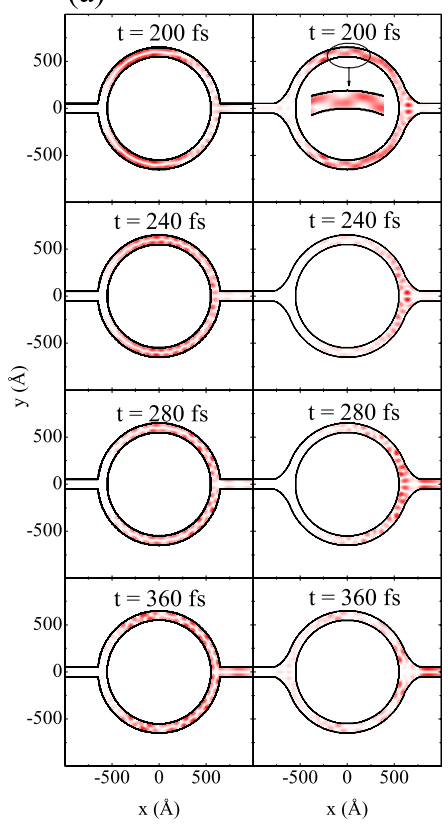

(b)

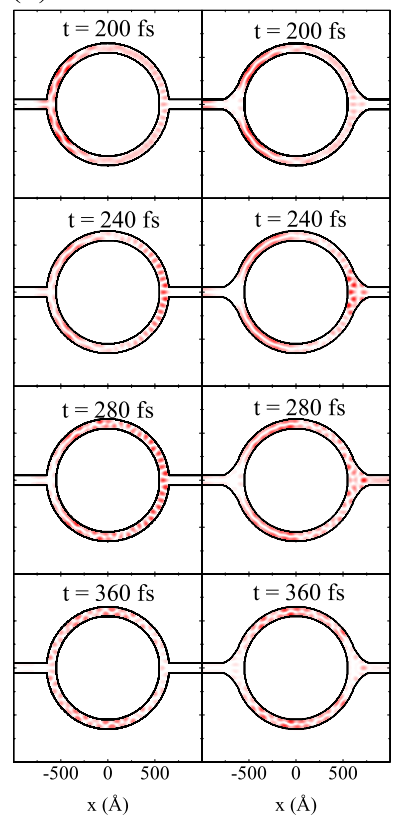

FIG. 12. (Color online) Contour plots of the time evolution of the electron density, for right-angle (left panels) and smooth (right panels) lead-ring connections, in the presence of an applied magnetic field $B=0.18 \mathrm{~T}$, perpendicular to the ring plane. The initial wave packet has width $\sigma=200 \AA$, energy $\varepsilon_{3}$ and is in the (a) lowest subband with wave vector $k_{3}^{(1)}$, and in the (b) second subband with wave vector $k_{3}^{(2)}$. 


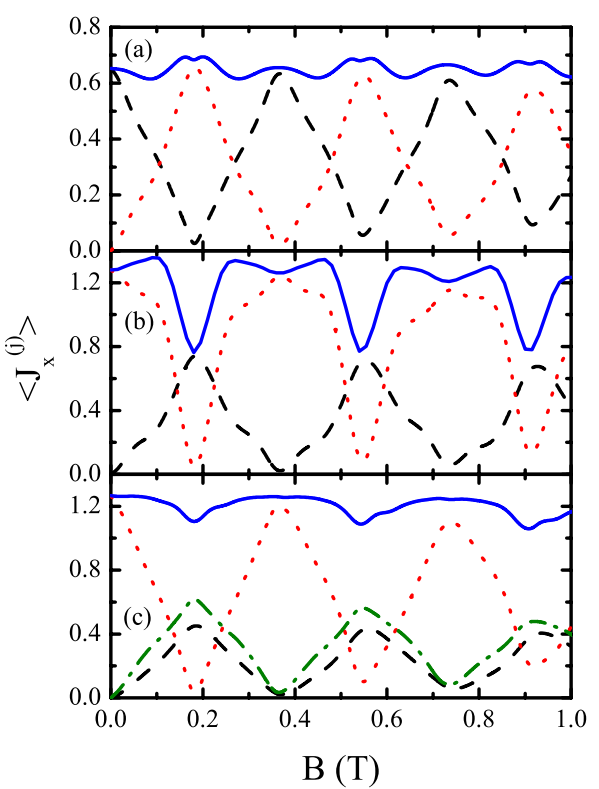

FIG. 13. (Color online) Contributions $\left\langle J_{x}^{(j)}\right\rangle$ of the ground (black, dashed), first-excited (red, dotted), and second-excited (green, dashed-dotted) subband states to the transmission current, as functions of the magnetic field $B$, calculated at the right channel, considering wave packets with energy $\varepsilon_{3}$ in the (a) first and (b) second subband, and (c) with energy $\varepsilon=430 \mathrm{meV}$ in the second subband. The blue solid line is the sum of the contributions due to the three subband states, $\left\langle J_{x}^{(1)}\right\rangle+\left\langle J_{x}^{(2)}\right\rangle+\left\langle J_{x}^{(3)}\right\rangle$.

zero magnetic field the projection $P_{2}$ at the right channel for wave packets with energies $\varepsilon_{1}$ and $\varepsilon_{2}$ are also nonzero but they become strongly dominant over $P_{1}$ only for $\varepsilon_{2}$ and $\varepsilon_{3}$. Notice, however, that for $\varepsilon_{2}$, as well as for $\varepsilon_{1}$, the transmission probability at this magnetic field intensity is almost zero [see Fig. 10(a)] so that this effect is better observed for $\varepsilon_{3}$. As the magnetic field increases, $P_{2}\left(P_{1}\right)$ at the left channel undergoes just very small fluctuations, even for $\varepsilon_{3}$ in the first (second) subband.

The time-averaged contributions $\left\langle J_{x}^{(j)}\right\rangle$ of the states of the first (black, dashed), second (red, dotted), and third (green, dashed-dotted) subbands to the probability current are plotted in Fig. 13 as a function of the magnetic flux, for wave packets with energy $\varepsilon_{3}$ in the first (a) and second (b) subbands, and with energy $430 \mathrm{meV}$ in the second subband (c). Since in (a) and (b) the wave packet energy is much lower than the third subband edge, the contribution $\left\langle J_{x}^{(3)}\right\rangle$ of this state to the current is almost zero, then, it is not shown. The sum of $\left\langle J_{x}^{(j)}\right\rangle$ for these three subband states is shown as a blue solid line, which mimics the transmission probabilities shown in Fig. 10(a) for $\varepsilon_{3}$, in a qualitative way. In Fig. 13(a) we observe that, considering a wave packet of energy $\varepsilon_{3}$ in the first subband, for a magnetic field corresponding to a flux equal to an integer (half integer) of the quantum flux, the outgoing wave function on the right channel is predominantly in the first (second) subband. Similar conclusions can be drawn by analyzing the results in Figs. 13(b) and 13(c). In other words, these effects suggest that, besides the wellknown periodic $\mathrm{AB}$ oscillations in transmission probabilities, observed in Fig. 10, when we consider a wave packet with energy higher than the second subband edge, the projections of the outgoing wave packet over the subbands states in the right lead also present $\mathrm{AB}$ oscillations with period $\phi_{0}=h / e$. These results were obtained considering a right-angle leadring connection but similar effects are also observed for smooth junctions (not shown in this work).

It is worthy to point out that if one considers a strong magnetic field or a wide lead, a mixing of subband states is also expected in the leads because of the magnetic force, which can deflect the wave function and make it move in a zigzag trajectory along the channels. This effect could not be observed in previous theoretical works, ${ }^{9,10}$ where the traveling wave packet was described as a sequence of Gaussian basis functions centered along the same axis so that the magnetic force is not active in the leads and hence, the magnetic field could not deflect the wave function in the leads. However, for our finite width channels the magnetic force is active in the leads. Yet, for the weak magnetic fields we have considered so far, our channels are narrow enough to suppress the deflection of the wave function due to the magnetic field, and the period of a possible zigzag trajectory of the wave packet would be much longer than the leads extent.

\section{ASYMMETRIC RING: EFFECTS OF GAUSSIAN POTENTIALS AND IMPURITIES}

As discussed in Sec. III C, in the absence of a magnetic field and when the wave packet is injected in a single subband, after it passes through the first lead-ring junction, it is scattered to other accessible subband states. However, as it reaches the second lead-ring junction, a completely destructive interference occurs for these other subband states so that the wave function leaves the ring in the initial subband state. This scenario no longer holds when a magnetic field is present and the outgoing wave function can be distributed over several subband states in the right lead. Actually, for $\Phi=(n+1 / 2) \phi_{0}(n=$ integer $)$, the $\mathrm{AB}$ effect is responsible for a completely destructive interference of the initial subband state of the wave function, consequently, the backscattered wave function has no projection over this state and is therefore situated in the other subband states, as observed in Fig. 13. Any extra phase shift in the wave function when propagating in the arms of the ring will disturb the destructive interference at the second junction. Such an extra phase shift can be induced by a magnetic field or through an electric field, i.e., potential variations. In the latter case, it is called the electrostatic $\mathrm{AB}$ effect. As an example, we consider a Gaussian quantum-well potential that is localized in the arm of the ring

$$
\bar{V}(x, y)=-V_{G} \exp \left\{-\frac{1}{2 \sigma_{G}}\left[\left(x-x_{\mathrm{arm}}\right)^{2}+\left(y-y_{\mathrm{arm}}\right)^{2}\right]\right\} .
$$

First we take the potential in the middle of the upper arm of the ring, i.e., at $\left(x_{\mathrm{arm}}, y_{\mathrm{arm}}\right)=\left(0, R_{\mathrm{av}}\right)$. The transmission probabilities for this case are plotted as functions of the potential depth $V_{G}$ in Figs. 14(a) and 14(d), for rings with right-angle and smooth $\left(R_{S}=300 \AA\right)$ lead-ring connections, respectively, 

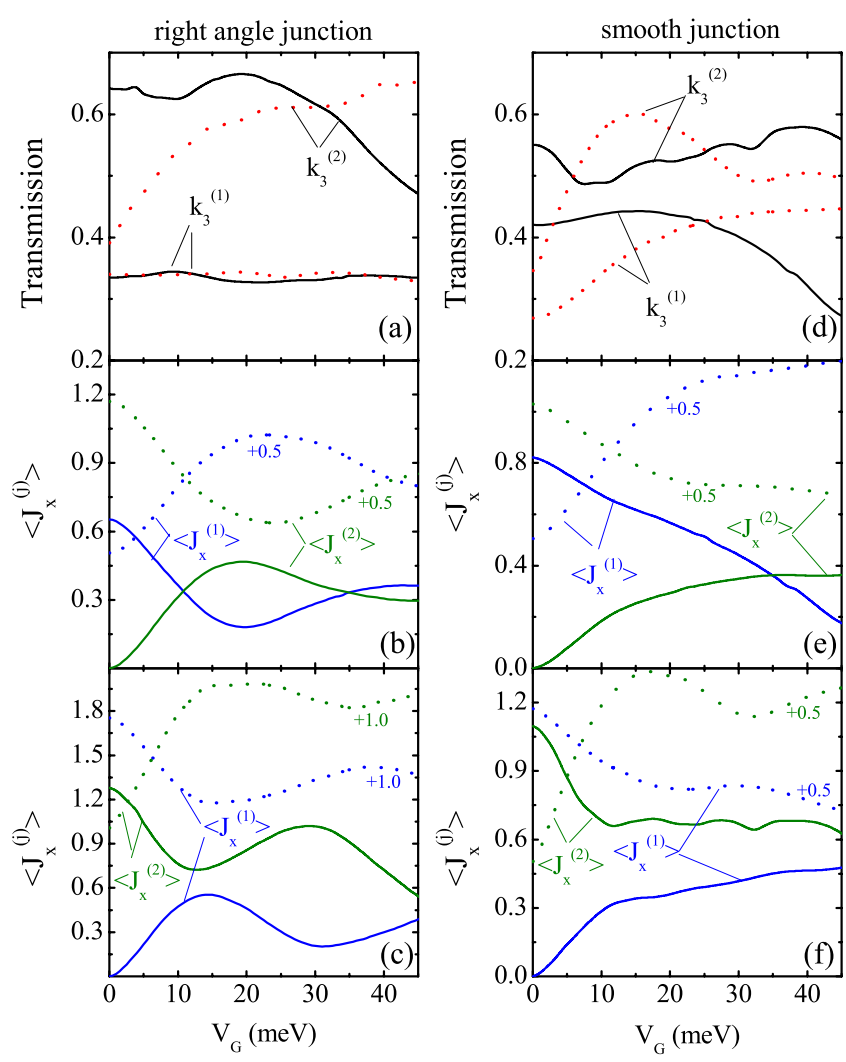

FIG. 14. (Color online) [(a),(d)] Transmission probability as a function of the depth $V_{G}$ of the Gaussian well, for wave packets with energy $\varepsilon_{3}$ in the first subband $\left(k_{3}^{(1)}\right)$ and in the second subband $\left(k_{3}^{(2)}\right)$, considering quantum rings with right-angle (left panels) and smooth, with $R_{S}=300 \AA$, (right panels) lead-ring connections. Two values of the magnetic field $B$ are considered: $0 \mathrm{~T}$ (solid) and 0.18 $\mathrm{T}$ (dotted). [(b),(e)] Time-averaged currents $\left\langle J_{x}^{(1)}\right\rangle$ and $\left\langle J_{x}^{(2)}\right\rangle$, of the first and second subbands, respectively, considering a wave packet initially in the first subband, with $k_{3}^{(1)}$. [(c),(f)] Time-averaged currents $\left\langle J_{x}^{(1)}\right\rangle$ and $\left\langle J_{x}^{(2)}\right\rangle$ for a wave packet initially in the second subband, with $k_{3}^{(2)}$. The lines $\left\langle J_{x}^{(1)}\right\rangle$ and $\left\langle J_{x}^{(2)}\right\rangle$ for $B=0.18 \mathrm{~T}$ (dotted) were shifted by +0.5 , for clarity [except in (c), where they were shifted by +1.0$]$.

considering wave packets with energy $\varepsilon_{3}$ initially in the first $\left(k_{3}^{(1)}\right)$ and second $\left(k_{3}^{(2)}\right)$ subband. Two values of magnetic field are considered, $B=0$ (solid, black) and $0.18 \mathrm{~T}$ (dotted, red), where the later corresponds to $\Phi=\phi_{0} / 2$. If the wave packet starts in the first subband, with $k_{3}^{(1)}$, for a system with right-angle connections, the phase shift due to the Gaussian well is not able to disturb too much the transmission probabilities, and their values for both values of the magnetic field are almost the same. However, for smooth junctions or for wave packets with wave vector $k_{3}^{(2)}$, the Gaussian well potential in one of the arms of the ring is responsible for oscillations in the transmission probability, as its depth $V_{G}$ increases. An analysis of the time-averaged currents $\left\langle J_{x}^{(1)}\right\rangle$ (blue) and $\left\langle J_{x}^{(2)}\right\rangle$ (green) propagating in the subband state $j$ $=1$ and $j=2$, respectively, as illustrated in Figs. 14(b) and 14(e), for $k_{3}^{(1)}$, and (c) and (f), for $k_{3}^{(2)}$, helps us to understand the behavior of the transmission probabilities. As observed in Fig. 14(b), for $B=0 \mathrm{~T}(0.18 \mathrm{~T})$, all the enhancement (reduction) in $\left\langle J_{x}^{(2)}\right\rangle$ as $V_{G}$ increases, is completely compensated by

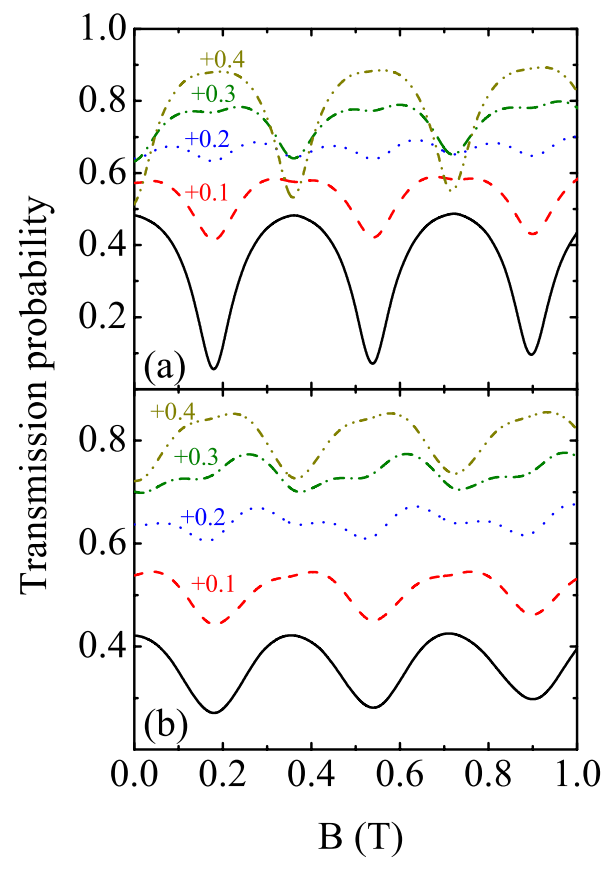

FIG. 15. (Color online) Transmission probability as a function of the magnetic field for wave packets with energy (a) $\varepsilon_{2}$ and (b) $\varepsilon_{3}$ in the first subband $\left(k_{3}^{(1)}\right)$, considering quantum rings with smooth $R_{s}=300 \AA$ lead-ring connections, for several values of depth $V_{G}$ of the Gaussian well: 0 (black, solid), 10 (red, dashed), 20 (blue, dotted), 30 (green, dashed-dotted), and $40 \mathrm{~V}$ (yellow, dashed-dotteddotted). The latter four curves are shifted in order to help visualization and the amount of shift is indicated on top of the curves.

a reduction (enhancement) in $\left\langle J_{x}^{(1)}\right\rangle$ so that the transmission probability remains almost the same for any $V_{G}$. This effect happens only for $\varepsilon_{3}$ in a structure with right-angle junction, which is a system where the $\mathrm{AB}$ oscillations in $T$ exhibit halved period and small amplitudes (cf. Fig. 10 blue solid curve). For all the other cases, such a compensation is not present, so that the curves for $B=0$ and $0.18 \mathrm{~T}$ are strongly dependent on $V_{G}$. In all cases, the phase shift between wave functions traveling through the upper and lower ring arms is responsible for disturbing the otherwise completely destructive interference for other subband states in the second leadring junction, allowing for an outgoing wave function which has projections over both subbands in the right channel when $V_{G} \neq 0$.

This effect can be verified using a similar experimental setup as in Ref. 7. By tuning the back gate voltage one can select electrons traveling with energy $\varepsilon_{1}, \varepsilon_{2}$, or $\varepsilon_{3}$ through the ring. The gate electrode localized over the center of one of the ring arms induces a localized potential that is tunable by the voltage applied on this gate. Besides, the experimental results of Ref. 7 suggest that the presence of an electrostatic potential in one of the ring arms induces a phase shift in the AB oscillations. The transmission probabilities calculated with our model for a smooth $\left(R_{s}=300 \AA\right)$ ring are shown as functions of the magnetic field in Fig. 15 for several values of $V_{G}$, considering incoming wave packets in the first subband with energies (a) $\varepsilon_{2}$ and (b) $\varepsilon_{3}$. In fact, a change in the position of the transmission minima occurs as $V_{G}$ increases from 0 (black, solid) to $40 \mathrm{~V}$ (yellow, dashed-dotted-dotted), 


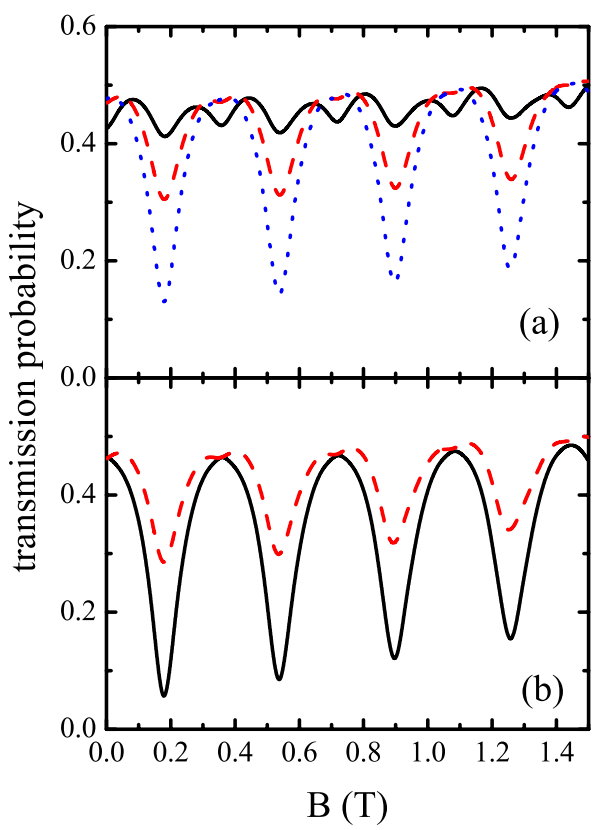

FIG. 16. (Color online) Transmission probabilities for a wave packet with energy $\varepsilon_{2}$, as functions of the magnetic field, considering smooth channel-ring connections, in the presence of (a) one impurity, localized at three different distances $z_{\text {imp1 }}$ from the ring plane: $1 \AA$ (black, solid), $100 \AA$ (red, dashed), and $400 \AA$ (blue, dotted), and (b) two impurities, each one localized symmetrically in one arm of the ring, at distances $z_{\text {imp } 1}=1 \AA$ and $z_{\text {imp } 2}=1 \AA$ (black, solid) or $z_{\text {imp } 2}=100 \AA$ (red, dashed).

which can be interpreted as a phase shift of $\pi$ in the AB oscillations. Actually, the results for intermediate values of $V_{G}$, namely, 10 (red, dashed), 20 (blue, dotted), and $30 \mathrm{~V}$ (green, dashed-dotted), show that the Gaussian potential is responsible for a reduction (increase) in the transmission probability at $\Phi=n \phi_{0}\left[\Phi=(n+1 / 2) \phi_{0}\right]$ so that the oscillations are shifted over $\phi_{0}$ when $V_{G}=40 \mathrm{~V}$. The reason for this flip in the $A B$ oscillations is owing to the fact that when the wave function hits a resonance of the potential well, it picks up a phase of $\pi$, which turns the $\mathrm{AB}$ maxima into minima and vice versa. ${ }^{9,10}$

Similarly, the influence of the presence of one negative impurity, localized at $\mathbf{r}_{\mathrm{imp}}=\left(0, R_{\mathrm{av}}, z_{\mathrm{imp}}\right)$ (i.e., in the middle of the upper arm of the ring, at a distance $z_{\text {imp }}$ from the ring plane) on the transmission $T$ and reflection $R$ probabilities is illustrated in Fig. 16(a), for a wave packet with energy $\varepsilon_{2}$. Smooth lead-ring connections are considered in Figs. 16(a) and $16(\mathrm{~b})$. If the impurity is close to the plane, at $z_{\text {imp }}$ $=1 \AA$ (black, solid), the AB oscillations are significantly affected; the period of such oscillations on the transmission and the reflection probabilities as a function of the magnetic field is halved. This behavior is similar to that found by Szafran and Peeters ${ }^{9}$ in mesoscopic one-dimensional rings, when a Gaussian potential is inserted in one of the ring arms. As the impurity is put far from the ring plane, the original period of $\mathrm{AB}$ oscillations is recovered, though the presence of the impurity still affects the peak-to-valley difference in these oscillations, as observed for $z_{\text {imp }}=100 \AA$ (red, dashed) and $400 \AA$ (blue, dotted), for instance. Actually, the presence of an impurity in only one of the ring arms strongly breaks the azimuthal symmetry of the system, which is decisive for $\mathrm{AB}$ oscillations. If one considers two impurities localized in diametrically opposite sides of the ring, as $\mathbf{r}_{\mathrm{imp} 1}$ $=\left(0, R_{\mathrm{av}}, z_{\mathrm{imp} 1}\right)$ and $\mathbf{r}_{\mathrm{imp} 2}=\left(0,-R_{\mathrm{av}}, z_{\mathrm{imp} 2}\right)$, for instance, the imbalance between the arms is removed and the original period of $\mathrm{AB}$ oscillations is recovered, as shown in Fig. 16(b), even if $z_{\text {imp1 }} \neq z_{\text {imp2 }}$ (red, dashed). The peak-to-valley difference is also almost recovered for $z_{\text {imp } 1}=z_{\text {imp2 }}$, even when the impurities are very close to the ring plane, as can be observed if one compares the results for $z_{\mathrm{imp} 1}=z_{\mathrm{imp} 2}=1 \AA$ (black, solid) with those for $\varepsilon_{2}$ in smooth channel-ring systems in the absence of impurities [see Fig. 10(b), red-dashed curve]. The recovering of $\mathrm{AB}$ oscillations when two impurities are localized in diametrically opposite sides of a quantum ring confirms the recent results of Farias et al. ${ }^{14}$ for the case of quantum rings without leads.

\section{CONCLUSIONS}

We presented a theoretical study of the time evolution of a Gaussian wave packet in semiconductor quantum rings and $\mathrm{T}$ wires, where we studied the effects due to smooth leadring connections, magnetic fields, the presence of a Gaussian potential or an impurity in the ring $\operatorname{arm}(\mathrm{s})$ on the transmission probabilities. Although one would intuitively expect that a smooth connection will lead to a higher transmission probability, our results show that this depends strongly on the wave packet energy since such connections act like confining potentials, where quantum resonances are present, the transmission probability is affected not only by the smoothness of the junction but also by the energy of the incoming wave packet.

In the absence of a magnetic field, for higher wave packet energies, the electron is scattered at the first lead-ring connection to other subbands states but remarkably it is scattered back to the initial subband at the second lead-ring junction so that the incoming and outgoing wave functions in the leads are always in the same subband. However, a magnetic field can influence strongly the interference at the second junction such that at the $\mathrm{AB}$ resonances we found complete destructive interference of only the initial subband state, which results in incoming and outgoing electrons ending up in different subbands. This effect strongly affects the transmission probabilities, changing the amplitude and the period of $\mathrm{AB}$ oscillations.

The effect of asymmetry is analyzed by considering a negative impurity or a Gaussian potential localized in one of the ring arms. In the presence of such an asymmetry, it is possible to half the period of the $\mathrm{AB}$ oscillations and to reduce its amplitude. However, if two negative impurities are localized in diametrically opposite sides of the ring, the symmetry of the system is recovered and so are the period and the amplitude of the $\mathrm{AB}$ oscillations.

\section{ACKNOWLEDGMENTS}

This work was financially supported by the Brazilian National Research Council (CNPq), under Contract No. Nano- 


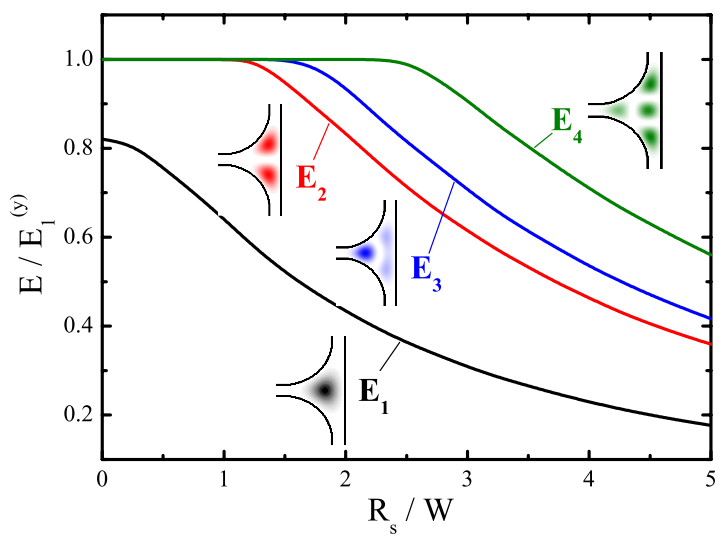

FIG. 17. (Color online) Energies of four low-lying bound states of a T-wire junction [see Fig. 1(b)], as functions of the radius of the circle describing the smooth junction. The insets show the probability density of the four bound states in case of $R_{S} / W=4$.

BioEstruturas 555183/2005-0, Fundação Cearense de Apoio ao Desenvolvimento Científico e Tecnológico (Funcap), CAPES, Pronex/CNPq/Funcap, the Bilateral program between Flanders and Brazil, and the Belgian Science Policy (IAP).

\section{APPENDIX: LOCALIZED STATES AND TIME EVOLUTION IN T WIRES}

To understand better the importance of the lead-ring connection on the transmission, we study here the T-shaped wire structure. This structure, as shown in Fig. 1(b), is formed by a junction of two perpendicular quantum wells. Classically, this system exhibits unbound motion but it has been shown that this geometry can support one quantum bound state. ${ }^{13}$ Other systems with classical unbound motion, such as crossshaped potentials, bend wires, and quantum cavities with channels, have been studied previously, ${ }^{22}$ where quantum bound states were also found. The origin of these quantum bound states lies in the fact that the quantum wells forming the channels are responsible for a discrete energy spectrum in these regions, with nonzero ground-state energy. Therefore, in the intersection between the channels, the electron can occupy a state which has an energy lower than the zeropoint energy in the leads and, hence, it will not be able to propagate through the leads.

In Fig. 17, the eigenenergies for $\mathrm{T}$ wires with smooth connections are shown as functions of the radius $R_{s}$ of the circle describing the connection, considering channels of width $W=100 \AA$. The eigenstate energies are divided by the ground-state energy $E_{1}^{(y)}$ of the channels while the radii $R_{s}$ are divided by the channel width $W$. Notice that, for a simple connection, i.e., for $R_{s}=0 \AA$, only one confined state can be found, but, as $R_{s}$ increases, more states are localized at the junction. The insets illustrate the squared modulus of the wave functions for each confined state when $R_{s}=400 \AA$. Results of Fig. 17 for T-shaped wires can be compared to the results for the $W=100 \AA$ lead-ring system, studied in Sec. III A. For a right-angle connection, there is only one bound state in both systems, which has energy $E_{0}=44(46) \mathrm{meV}$ for
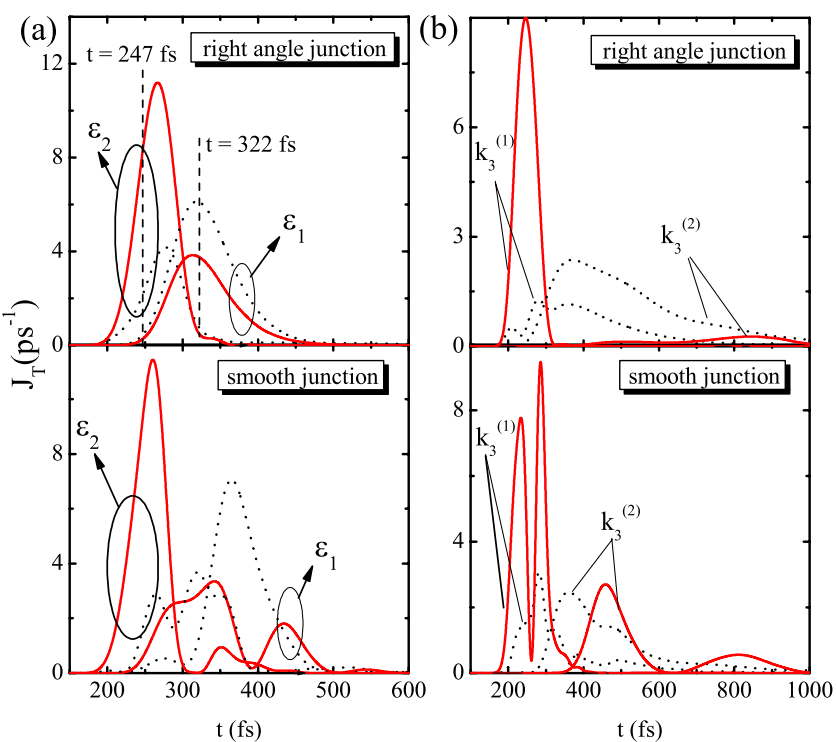

FIG. 18. (Color online) Time-dependent probability current, calculated at the vertical (black, dotted) and horizontal (red, solid) leads of a $W=100 \AA$ T-shaped wire, at a distance $1400 \AA$ from the center of the junction, considering right-angle and smooth, with $R_{S}=300 \AA$, connections. In (a), two values of wave packet energy were considered, $\varepsilon_{1}$ and $\varepsilon_{2}$ while in (b), we consider $\varepsilon_{3}$ in subbands $k_{3}^{(1)}$ and $k_{3}^{(2)}$.

the T-wire (lead-ring) case. The small difference in energy is a consequence of the bending of the ring arms at the junction. For a smooth connection, with $R_{s}=300 \AA$, the T-shaped wire has four bound states, with energies $E_{0} \simeq 16 \mathrm{meV}, E_{1}$ $\simeq 33 \mathrm{meV}, E_{2} \simeq 38 \mathrm{meV}$, and $E_{3} \simeq 48 \mathrm{meV}$ whereas the lead-ring system presents two pairs of degenerated states, with energies $E_{0}=E_{1} \simeq 26 \mathrm{meV}$ and $E_{2}=E_{3} \simeq 50 \mathrm{meV}$. In this case, the difference in energies is a consequence of the fact that for a smooth lead-ring junction the effective area where the bound state can reside is smaller than in the case of a smooth $\mathrm{T}$ junction.

T-shaped wires has attracted much interest also because these structures provide the possibility of constructing transistors acting by quantum modulation. The transport properties of T-shaped wires was theoretically studied by Sols et al. ${ }^{23}$ where they calculated the transmission probability as a function of the electron energy and effective length of the lead perpendicular to the electrons motion for straight junctions. In Fig. 18, the time-dependent probability current is calculated at the horizontal (red, solid) and vertical (black, dotted) leads, at a distance of $1400 \AA$ from the center of the junction. The probability current in the horizontal (vertical) lead is related to the reflected (transmitted) part of the wave packet. The initial wave packet starts in the horizontal lead, at $x_{0}=-1100 \AA$. For a right-angle connection, sharp peaks for transmitted (dotted) and reflected (solid) packets are observed in Fig. 18(a) for $\varepsilon_{1}$ and $\varepsilon_{2}$. Since the distance from the junction is the same for the points where $J_{T}$ is computed in vertical or horizontal leads, both peaks were expected to present maximum values at the same time. However, a small time delay of about $6 \mathrm{fs}$, between the transmitted and reflected maxima is observed. Besides, the time for a classical particle with energy $\varepsilon_{1}\left(\varepsilon_{2}\right)$ traveling through the same dis- 


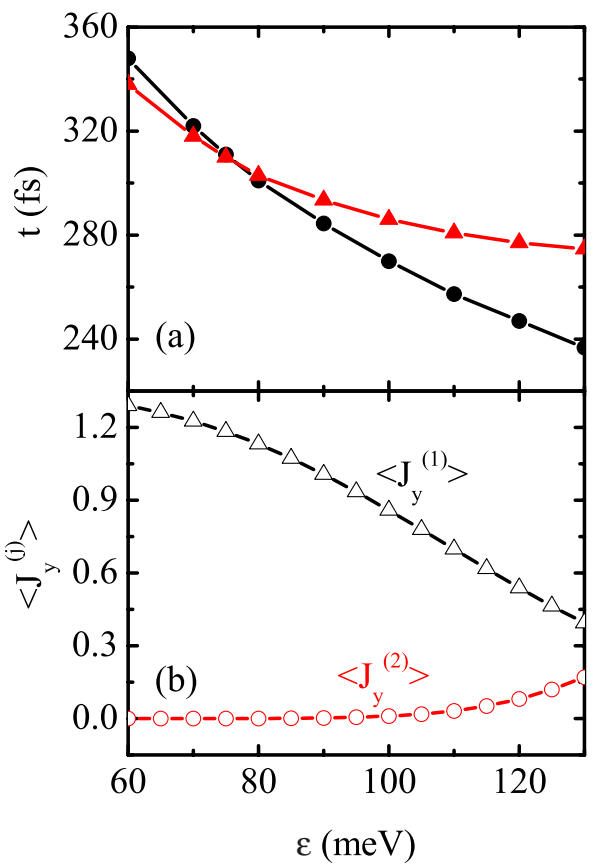

FIG. 19. (Color online) (a) Calculated transmission times for a classical particle traveling in a T-shaped wire (black circles) and the time where the maximum value of $J_{T}$ occur in the vertical lead (red triangles), as functions of the wave packet energy $\varepsilon$, for leads of $W=100 \AA$ width, considering right-angle connections. (b) Timeaveraged currents in the first $\left(\left\langle J_{x}^{(1)}\right\rangle\right.$, black $)$ and second $\left(\left\langle J_{x}^{(2)}\right\rangle\right.$, red $)$ subbands.

tances is about 322 (247) fs whereas the times where the peaks of transmitted and reflected waves occur are both lower (higher). The times for the classical approximation are shown as vertical dashed lines in Fig. 18 (top), for comparison. Actually, lower (higher) values for classical times, for a particle with energy $\varepsilon_{1}\left(\varepsilon_{2}\right)$, were found also for the lead-ring system, as mentioned in Sec. III B. A comparison between the times where the maxima of the transmission peaks occur (red triangles) and the transmission times for classical particles (black circles), as functions of the wave packet energy, in a $W=100 \AA \mathrm{T}$ wire, is shown in Fig. 19(a). It is observed that, for energies higher than $\sim 75 \mathrm{meV}$, the classical approach underestimates the transmission time in these systems. Actually, as the energy increases, the projection of the transmitted wave packet over the second subband state becomes larger, as observed in Fig. 19(b) and since the wave vector $k$ for this subband is lower than that of the first subband, for the same energy, the wave function slows down, which explains the higher transmission time, as the classical approach accounts only for the first subband state. For a smooth connection with $R_{s}=300 \AA$, as well as for energy $\varepsilon_{3}$, due to interferences at the junction, the outgoing waves do not yield sharp peaks in $J_{T}$, thus it becomes difficult to discuss the problem in comparison with a classical traveling particle in this case.

The transmission probability $T$ as a function of the wave packet energy $\varepsilon$ for a T-shaped wire with leads of width $W$ $=100 \AA$ is shown in Fig. 20(a), for several values of the radius $R_{S}$ of the circle describing the smoothness of the junc-
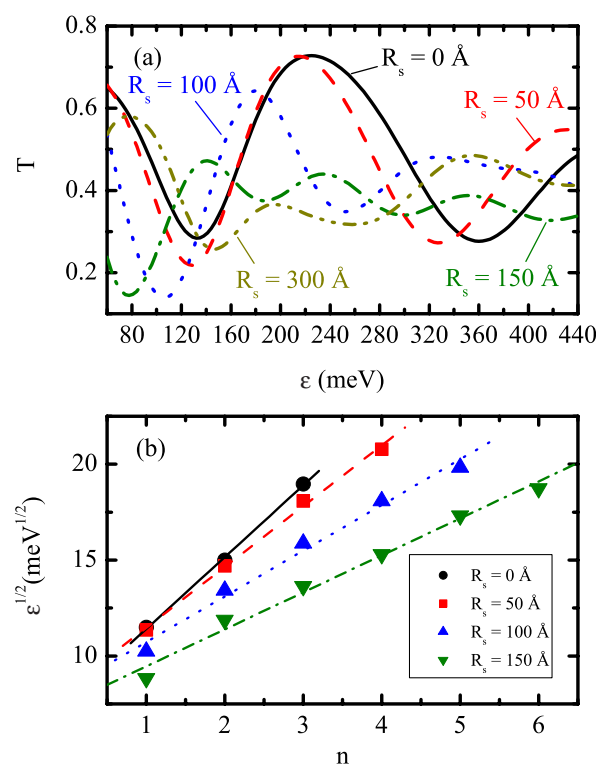

FIG. 20. (Color online) (a) Transmission probability as a function of the wave packet energy $\varepsilon$ (in the lowest subband) for a T-shaped wire with leads of $W=100 \AA$, considering several values of radius $R_{s}$ for smooth connections. (b) Square root of the energies of the peaks and valleys for each curve shown in (a). The straight lines are linear fits to the symbols. Similar types of curves in (a) and (b) correspond to the same $R_{s}$.

tion. In Fig. 10, if one compares the results for (a) rightangle and (b) $R_{s}=300 \AA$ lead-ring connections, one observes that, for $\varepsilon_{1}\left(\varepsilon_{2}\right)$, the presence of smooth connections reduces (increases) the transmission probability. This is also observed for the T-wire case, if we analyze the results for right angle (black solid) and $R_{s}=300 \AA$ (yellow dashed-dotted-dotted), for energies $\varepsilon=70$ and $120 \mathrm{meV}$. Moreover, the transmission probability as a function of the wave packet energy exhibits an oscillatory behavior, which strongly depends on the radius $R_{s}$ of the smooth connection. This oscillatory behavior resembles the one observed in the transmission coefficients for a one-dimensional quantum-well structure, when one considers electron energies which are higher than the barriers height. Indeed, our T-wire structure can be interpreted as a system consisting of two barriers, created by the zero-point energy of the channels, and a well, localized at the junction so that the incoming wave packet in the horizontal channel has energies which are, by definition, higher than these barrier heights. For a one-dimensional square well of width $L$ and considering electrons with energy higher than the barrier height, a maximum of transmission probability is obtained when half the wavelength fits the well width $L$, i.e., $n \lambda / 2$ $=L$, resulting in the resonant energy

$$
\varepsilon_{n}=\frac{\hbar^{2}}{2 m_{e}}\left(\frac{n \pi}{L}\right)^{2},
$$

for the $n$th transmission peak. From this expression, it is expected that $\sqrt{\varepsilon_{n}}$ increases linearly with $n$.

Such a linear trend is observed in the results for $\mathrm{T}$ wires, as shown in Fig. 20(b), where the square root of the wave packet energies $\varepsilon^{1 / 2}$ for the $n$th maximum or minimum of 
transmission probability, observed in Fig. 20(a), are plotted as symbols, for several values of radius $R_{s}$ of the smooth connection between leads. Actually, the analogy between our T-wire system and a one-dimensional square well is of course not perfect, specially when smooth connections are considered. Also, we are dealing with a wave packet, not with a simple plane wave, and the resonances obtained by means of Eq. (A1) for a square well are not expected to be the same as for a Gaussian wave packet. Nevertheless, the linear fits of the symbols in Fig. 20(b), which are shown as curves, are an interesting tool to understand qualitatively the behavior of transmission probabilities in $\mathrm{T}$ wires, in compari- son to a one-dimensional well. From these linear fits, one can estimate the effective width of the confinement potential of the T-wire junction in this quantum-well picture. The slopes $s$ of the $\varepsilon^{1 / 2} x n$ curves can be obtained from Eq. (A1), for a square well, as $s=\sqrt{\hbar^{2} / 2 m} \pi / L$. For $R_{s}=0$ (black, solid), 50 (red, dashed), 100 (blue, dotted), and 150 (green, dasheddotted) in the T-wire system, the slopes of the lines shown in Fig. 20(b) correspond to one-dimensional quantum-well widths $L$ of $\sim 255.9,302.4,402.0$, and $496.5 \AA$, respectively. The increase in $L$ with $R_{s}$ is intuitively expected because of the increasing area of the connection with $R_{s}$.

\footnotetext{
*andrey@fisica.ufc.br

†gil@fisica.ufc.br

*francois.peeters@ua.ac.be

${ }^{1}$ Y. Aharonov and D. Bohm, Phys. Rev. 115, 485 (1959).

${ }^{2}$ R. A. Webb, S. Washburn, C. P. Umbach, and R. B. Laibowitz, Phys. Rev. Lett. 54, 2696 (1985).

${ }^{3}$ G. Timp, A. M. Chang, J. E. Cunningham, T. Y. Chang, P. Mankiewich, R. Behringer, and R. E. Howard, Phys. Rev. Lett. 58, 2814 (1987).

${ }^{4}$ B. Hackens, F. Martins, T. Ouisse, H. Sellier, S. Bollaert, X. Wallart, A. Cappy, J. Chevrier, V. Bayot, and S. Huant, Nat. Phys. 2, 826 (2006).

${ }^{5}$ F. Martins, B. Hackens, M. G. Pala, T. Ouisse, H. Sellier, X. Wallart, S. Bollaert, A. Cappy, J. Chevrier, V. Bayot, and S. Huant, Phys. Rev. Lett. 99, 136807 (2007).

${ }^{6}$ M. G. Pala, B. Hackens, F. Martins, H. Sellier, V. Bayot, S. Huant, and T. Ouisse, Phys. Rev. B 77, 125310 (2008).

${ }^{7}$ W. G. van der Wiel, Yu. V. Nazarov, S. De Franceschi, T. Fujisawa, J. M. Elzerman, E. W. G. M. Huizeling, S. Tarucha, and L. P. Kouwenhoven, Phys. Rev. B 67, 033307 (2003).

${ }^{8}$ S. Gustavsson, R. Leturcq, M. Studer, T. Ihn, K. Ensslin, D. C. Driscoll, and A. C. Gossard, Nano Lett. 8, 2547 (2008).

${ }^{9}$ B. Szafran and F. M. Peeters, Phys. Rev. B 72, 165301 (2005).

${ }^{10}$ B. Szafran and F. M. Peeters, Europhys. Lett. 70, 810 (2005).
}

${ }^{11}$ M. Suzuki, Phys. Lett. A 146, 319 (1990).

${ }^{12}$ N. Watanabe and M. Tsukada, Phys. Rev. E 62, 2914 (2000).

${ }^{13}$ M. H. Degani, Phys. Rev. B 66, 233306 (2002).

${ }^{14}$ G. A. Farias, M. H. Degani, J. A. K. Freire, J. Costa e Silva, and R. Ferreira, Phys. Rev. B 77, 085316 (2008).

${ }^{15}$ A. Chaves, J. Costa e Silva, J. A. K. Freire, and G. A. Farias, Microelectron. J. 39, 455 (2008).

${ }^{16}$ R. Kosloff and D. Kosloff, J. Comput. Phys. 63, 363 (1986).

${ }^{17}$ D. Neuhasuer and M. Baer, J. Chem. Phys. 90, 4351 (1989).

${ }^{18}$ S. Mahapatra and N. Sathyamurthy, J. Chem. Soc., Faraday Trans. 93, 773 (1997).

${ }^{19}$ A. Arnold, M. Ehrhardt, and I. Sofronov, Commun. Math. Sci. 1, 501 (2003).

${ }^{20}$ D. E. Manolopoulos, J. Chem. Phys. 117, 9552 (2002).

${ }^{21}$ G. Stefanucci and C.-O. Almbladh, Phys. Rev. B 69, 195318 (2004); V. Moldoveanu, V. Gudmundsson, and A. Manolescu, ibid. 76, 085330 (2007); A. Stan, N. E. Dahlen, and R. van Leeuwen, J. Chem. Phys. 130, 224101 (2009).

${ }^{22}$ F. M. Peeters, Science and Engineering of One- and ZeroDimensional Semiconductors, edited by S. P. Beaumont and C. M. Sotomayor Torres (Plenum, New York, 1990), p. 107.

${ }^{23}$ F. Sols, M. Macucci, U. Ravaioli, and K. Hess, Appl. Phys. Lett. 54, 350 (1989). 